\title{
The impact of cognitive skills on the distribution of the black-white wage gap
}

\author{
Donal O’Neill, ${ }^{\mathrm{a}, *}$, Olive Sweetman ${ }^{\mathrm{a}}$, Dirk Van de gaer $^{\mathrm{b}}$ \\ ${ }^{a}$ Economics Dept., National University of Ireland Maynooth, Maynooth, Co. Kildare, \\ Ireland and Research Associate NIRSA, NUI Maynooth, Ireland \\ ${ }^{\mathrm{b}}$ Ghent University, Hoveniersberg 24, B-9000, Gent, Belgium
}

Received 1 August 2003; received in revised form 1 January 2004; accepted 26 August 2004

\begin{abstract}
In this paper we use a semi-parametric estimation procedure to examine differences in the distribution of wages for black and white male workers in the US. In keeping with recent studies we find that differences in cognitive skills are an important determinant of the black-white wage gap and can explain almost the entire male racial wage gap among high wage workers. However, we find that equalising the distribution of cognitive skills will be less successful in reducing this gap at the lower end of the distribution.
\end{abstract}

(C) 2004 Elsevier B.V. All rights reserved.

JEL classification: C14; J15; J31; J71

Keywords: Wage Distribution; Black-White Wage Gap; Semi-Parametric Estimation

\section{Introduction}

Several studies have examined the mean wage gap between black and white workers in the US ${ }^{1}$. While looking at mean wages is useful it tells us nothing about what is happening at other parts of the distribution. For instance we may be particularly interested in

\footnotetext{
* Corresponding author. Tel.: +353 1 7083555; fax: +3531 7083934.

E-mail address: donal.oneill@may.ie (D. O’Neill).

${ }^{1}$ For a review of these studies see Altonji and Blank (1999). 
comparing the extent of low pay among black and white workers. Studies that focus on average wages offer no insight into this. We might also be interested in analysing the impact of a policy change on a particular part of the wage distribution. This requires altering the traditional decomposition procedures developed by Blinder (1973) and Oaxaca (1973) in order to understand how the entire wage distribution changes as result of a proposed policy. $^{2}$

In a recent study DiNardo et al. (1996) developed a semi-parametric procedure for estimating counterfactual distributions. They used this procedure to account for changes in wage inequality over time. In this paper we use this approach to re-examine the blackwhite wage gap for male workers in the US. ${ }^{3}$ In particular we focus on recent work by Neal and Johnson (1996) who argue that 'one test score explains ...much of the [blackwhite wage] gap for young men.' They attribute the difference in test scores to pre-labor market forces such as family background and school quality. They conclude that the disadvantages facing young black workers today arise from obstacles they faced as children in acquiring human capital and that public policy should focus on the plight of black children in acquiring skills that are valued in the labor market. These results and policy conclusions have been the subject of debate. Some economists have criticised both the Neal and Johnson approach and their conclusions. Darity and Mason (1998) raise questions as to how one should interpret the test score variable used by Neal and Johnson. They note that when test scores are adjusted for post-adolescent choice variables such as schooling, along the lines suggested by Rodgers and Spriggs (1996), they no longer explain the black-white wage difference. In contrast, Heckman (1998) reiterates the significance of the Neal and Johnson findings. He argues that "the objections raised by Darity and Mason against the Neal-Johnson study are largely specious" and points out that adjusting the test score as suggested above "misses the point of the Neal and Johnson article". In this paper we maintain the Neal and Johnson approach and use test scores to measure cognitive skills. However we extend their analysis by focusing on the distribution of the racial wage gap. Our distributional analysis shows that although adjusting for prelabour market skill differences can explain the average racial wage gap, equalization of these skills between black and white workers may not eliminate the wage gap at the lower end of the distribution.

\section{Estimating counterfactual distributions}

The traditional Blinder-Oaxaca wage decomposition involves estimating the wage that the average black worker would receive if he were given the characteristics of the average white worker but continued to be paid according to the black wage structure. To estimate this counterfactual the approach uses the fact that the ordinary least squares regression

\footnotetext{
${ }^{2}$ Roemer (1998) has suggested that a comparison of the wage gap between groups at various percentiles of their respective wage distributions may also help in understanding the degree of inequality of opportunity that exists among these individuals.

${ }^{3}$ For alternative procedures for analysing the distribution of the wage gap see Jenkins (1994), Gardeazabal and Ugidos (2004) and Bonjour and Gerfin (2001).
} 
passes through the sample mean of the data. In this paper we use the semi-parametric estimator developed by DiNardo et al. (1996) to estimate a similar counterfactual for the entire distribution, not just at the mean. This approach allows us to analyse the effect of cognitive skills on the entire distribution of wages. It also avoids making parametric assumptions that may result in incorrect inferences even for the average wage gap (Barsky et al. (2002)).

To simplify the exposition, assume that wages depend only on a composite measure of cognitive skill, which we denote by $t .{ }^{4}$ Each individual observation can be viewed as a vector $(w, t, R)$, consisting of a log wage $w$, a level of cognitive skill $t$, and an indicator for race $R$ which is $B$ or $W$. The observed density of wages for white workers can be written as:

$$
f_{W W}(w)=\int f_{W}(w, t) d t=\int f_{W}(w \mid t) f_{W}(t) d t
$$

where $f_{W}(w \mid t)$ is the density of log wages conditional on skill for white workers and $f_{W}(t)$ is the density of skills for this group. Likewise, the observed density of log wages for black workers is given by :

$$
f_{B B}(w)=\int f_{B}(w \mid t) f_{B}(t) d t
$$

By analogy with the Blinder-Oaxaca decomposition, we are interested in the density of wages for workers paid according to the black wage structure and like blacks in all respects except that they have the white distribution of test scores. We denote this counterfactual by $f_{B W}(w)$. If we assume that changing the skill distribution for black workers does not alter the black wage structure, $f_{B}(w \mid t)$, then it follows that the counterfactual can be written as

$$
f_{B W}(w)=\int f_{B}(w \mid t) f_{W}(t) d t
$$

We can now consider decomposing the difference in the wage distributions, $\left[f_{W W}(w)-\right.$ $\left.f_{B B}(w)\right]$, as follows:

$$
f_{W W}(w)-f_{B B}(w) \equiv\left[f_{W W}(w)-f_{B W}(w)\right]+\left[f_{B W}(w)-f_{B B}(w)\right]
$$

Using Eqs. (1)-(3) we can rewrite the components on the right hand side of Eq. (4) as:

$$
\begin{aligned}
& f_{W W}(w)-f_{B W}(w)=\int\left[f_{W}(w \mid t)-f_{B}(w \mid t)\right] f_{W}(t) d t \\
& f_{B W}(w)-f_{B B}(w)=\int f_{B}(w \mid t)\left[f_{W}(t)-f_{B}(t)\right] d t
\end{aligned}
$$

Eq. (6) shows that the difference between $f_{B W}(w)$ and $f_{B B}(w)$ is entirely due to differences in the distribution of skills. If this accounted for all of the difference between the black and white wage distributions we would have that $\left[f_{W W}(w)-f_{B B}(w)\right]=\left[f_{B W}(w)-\right.$

\footnotetext{
${ }^{4}$ Although we treat $t$ as a scalar the same methodology can be used when $t$ is a vector of characteristics.
} 
$\left.f_{B B}(w)\right]$ and $f_{B W}(\mathrm{w})=f_{W W}(w)$. Eq. (5) captures differences in the distribution of wages evaluated using the white distribution of skills. These differences may reflect either discrimination or differences in characteristics other than skills.

This approach can be used to decompose the log wage gap at different percentiles of the wage distributions. For example, the wage gap between the worker at the $p^{\text {th }}$ percentile of the black distribution and the corresponding worker in the white distribution can be written as:

$$
F_{W W}^{-1}(p)-F_{B B}^{-1}(p) \equiv\left[F_{W W}^{-1}(p)-F_{B W}^{-1}(p)\right]+\left[F_{B W}^{-1}(p)-F_{B B}^{-1}(p)\right]
$$

where $F_{R R^{\prime}}$ is the cumulative wage distribution associated with $f_{R R^{\prime}}$. As before, the second term on the right hand side of (7) measures the log wage gap resulting from differences in test scores and the first term captures any other remaining differences.

To implement this procedure we need to estimate the counterfactual density $f_{B W}(w)$. The key contribution of DiNardo et al. (1996) is to note that the required counterfactual can be written in terms of actual densities with the help of a reweighting function. To see this simply multiply and divide Eq. (3) by $f_{B}(w) . f_{B W}(w)$ can then be written as:

$$
f_{B W}(w)=\int f_{B}(w \mid t) f_{B}(t) \psi(t) d t
$$

where $\psi(t)$ is a re-weighting function, defined as $f_{W}(t) / f_{B}(t)$. From Eq. (8) we can see that the counterfactual wage density is the black density adjusted by an appropriate weighting function. Eq. (8) suggests that the required counterfactual can be obtained by reweighting the observed black wage density. The weights are chosen so that skill levels at which there are relatively more white workers receive a higher weight in the estimation of the density. Once we have obtained an estimate of the weighting function, $\hat{\psi}(t)$, it can be used to estimate the counterfactual density by weighted kernel methods. ${ }^{5}$ Specifically,

$$
\hat{f}_{B W}(w)=\frac{1}{n_{B}} \sum_{i \varepsilon N_{B}} \frac{1}{h} \hat{\psi}\left(t_{i}\right) K\left(\frac{w-W_{i}}{h}\right)
$$

where $N_{B}$ is the set of black individuals in our sample, $n_{B}$ is the number of black workers, $K($.$) is the kernel function, h$ is the bandwidth ${ }^{6}$ and $\hat{\psi}\left(t_{i}\right)$ is an estimate of the reweighting function. $^{7}$

Eq. (9) highlights the importance of the estimated reweighting function $\left(\hat{\psi}\left(t_{i}\right)=\hat{f}_{W}\left(t_{i}\right) /\right.$ $\left.\hat{f}_{B}\left(t_{i}\right)\right)$ for this approach. There are a number of possible ways of constructing $\hat{\psi}\left(t_{i}\right)$. One could adopt a nonparametric approach by simply grouping the sample according to $t$ and calculating the relative proportions of black and white individuals in each cell. Alternatively one could extend this approach by computing $\hat{\psi}\left(t_{i}\right)$ on the basis of kernel estimates of $f_{W}(t)$ and $f_{B}(t)$. The drawback with both of these approaches is that they suffer

\footnotetext{
${ }^{5}$ For a summary of kernel density estimators see DiNardo and Tobias (2001).

${ }^{6}$ Throughout this paper we assume a Gaussian Kernel and the bandwidth is chosen using the optimal bandwidth for the normal density-see, e.g., Silverman (1986) page 45. We have also estimated the densities using alternative bandwidths and this did not alter our results.

${ }^{7}$ In practice the individual weights are normalised by the mean of the weights to ensure that the density integrates to 1 .
} 
from the so-called curse of dimensionality. Once we extend the number of characteristics that we wish to control for, the cell-sizes for these estimators become very small. An alternative approach that overcomes this problem is to estimate the probabilities using a discrete choice model. To see this, rewrite $\psi(t)$ using Bayes' Rule as :

$$
\psi(t)=f_{W}(t) / f_{B}(t) \equiv\left[n_{B} / n_{W}\right] *[f(W \mid t) / f(B \mid t)]
$$

The first term in (10) is simply the ratio of black to white workers in the sample. The second term is the ratio of the probabilities that a worker is of a given race (black or white) conditional on $t$. This can be easily estimated using a discrete choice model such as a probit, where the dependent variable is a zero-one indicator variable for race and the independent variable is $t$. The advantage of this approach is that one can easily control for several characteristics by simply adding to the independent variables in the probit specification. This is the approach that we follow in the remainder of the paper. ${ }^{8}$ Once an estimate of $\hat{\psi}\left(t_{i}\right)$ is obtained, the required counterfactual density, $\hat{f}_{B W}(w)$, is estimated using the weighted kernel approach specified in Eq. (9).

\section{Data}

To estimate these distributions we use data from the National Longitudinal Surveys of Youth (NLSY79). This is the data set used by Neal and Johnson and is a panel data set that follows 12,686 youths born between 1957 and 1964. Given the narrow age range in these data we are constrained to focus on log wage differences over a limited part of the lifecycle. The wage data we use are for 1993. Retention rates in the NLSY79 were very high through 1993, remaining stable at about 90\% (Pergamit et al. (2001)). As well as the initial cross-sectional samples, the NLSY also contains supplementary samples that oversample all black youths and economically disadvantaged white youths. In our analysis we combine the cross-sectional and supplemental samples for black youths but use only the cross-sectional sample for whites. This procedure ensures that our resulting samples are representative of both the black and white populations. We follow Neal and Johnson by dropping observations for which the hourly wage is less than $\$ 1$ or greater than $\$ 75$ and by measuring cognitive skill using scores from the Armed Forces Qualification Test (AFQT). The AFQT is part of a battery of tests used by the military for enlistment, screening and job assignment. The tests are used to measure basic numeracy and literacy skills and were administered to almost all of the NLSY sample, irrespective of whether or not these individuals intended to apply to the military. In 1991 the National Academy of Sciences completed a study of the validity of the AFQT in measuring work performance, with special emphasis on racial bias, and found no evidence to suggest that the AFQT underpredicts productivity or job performance for black workers. This evidence suggests that the AFQT provides a racially unbiased predictor of basic skills. ${ }^{9}$ To allow us to

\footnotetext{
${ }^{8}$ For counterfactuals in which the number of conditioning variables is small we also estimated the weights using the nonparametric procedure discussed earlier. These results were very similiar to those based on the probit model.

${ }^{9}$ For a more detailed discussion of this measure of cognitive skills see Cawley et al. (1997).
} 
Table 1

Summary statistics

\begin{tabular}{lllll}
\hline Variable Name & Blacks & Whites & Blacks & Whites \\
\hline Log Wage & 2.18 & 2.44 & 2.21 & 2.45 \\
Standardised AFQT & -.58 & .51 & -.46 & .55 \\
Father's Highest Grade Completed & & & 10.39 & 12.42 \\
Rotter Scale & & 9.32 & 9.17 \\
Rosenberg Scale & 467 & 903 & 26.38 & 26.92 \\
$\mathrm{~N}$ & 4 & 316 & 840 \\
\hline
\end{tabular}

compare our results with those of Neal and Johnson we restrict our sample to workers who were aged 18 or younger when they took the test. We focus only on male workers. We adjust the test scores for remaining age effects and standardize the adjusted test scores so as to have mean zero and variance one.

In the final part of the paper we include an additional control for differences in family background, which we proxy using father's education. We also control for differences in non-cognitive skills, which we measure using an abbreviated version of the Rotter Locus of Control Scale developed by Rotter (1966) and the Rosenberg Self-Esteem Scale (Rosenberg (1965)). The Rotter scale is designed to measure the extent to which individuals believe they have control over their lives through self-motivation and selfdetermination. The scale ranges between 0 and 16 with higher values corresponding to a belief that the respondent has less control over their life. The Rosenberg scale is a 10 item scale which measures an individual's degree of approval or disapproval towards one-self. Individuals are given 10 statements of self-approval with which they are asked to strongly agree, agree, disagree or strongly disagree. Higher scores reflect higher levels of selfesteem. Previous research has shown that both these measures of non-cognitive skills have a significant impact on labour market outcomes. ${ }^{10}$ The final sample for the analysis based on AFQT scores contains 903 whites and 467 blacks. When the measures of father's education and non-cognitive skills are included in the analysis the sample sizes fall to 840 whites and 316 blacks. Summary statistics for the variables in these samples are given in Table 1.

\section{Results}

Neal and Johnson argue that controlling for differences in AFQT scores can explain a large proportion of the average black-white wage gap. Table 2 reproduces the central findings of their study for male workers. We see that the average log wage differential falls from .26 to .06 when we include a measure of cognitive skills. ${ }^{11}$ In this section of the paper we illustrate the distributional approach outlined earlier by examining the impact of cognitive skills on the entire distribution of log wages.

\footnotetext{
${ }^{10}$ See for example the discussion in Dunfion et al. (2001).

11 These results are slightly different than those of Neal and Johnson. They use pooled data from 1990 and 1991 while the wage data that we use refer to 1993 . Nevertheless the main conclusions from their analysis are still evident with these data.
} 
Table 2

Wage regressions (t-statistics in parentheses)

\begin{tabular}{lll}
\hline Dependent variable is Log weekly wage 1993 & & \\
\hline $\mathrm{n}=1370$ & $-.26(.03)$ & $-.06(.03)$ \\
Black & $.04(.01)$ & $.03(.01)$ \\
Age & & $.18(.01)$ \\
AFQT & .07 & $.01(.01)$ \\
AFQT $^{2}$ & .07 & .189 \\
$\mathrm{R}^{2}$ & & \\
\hline
\end{tabular}

Fig. 1 shows the log wage densities for black (bb) and white workers (ww). As noted earlier the mean wage of whites is approximately 26 percent higher than the mean black wage. We can also use the data from these densities to estimate the log wage gap at each centile of the wage distributions, the left hand side of (7). These results are presented in Fig. 2. We see that there is significant variation around the mean wage gap, which is indicated by the horizontal line at .26. At the low end of the distribution, the log wage gap is as low as .05. This may be attributable to the compression of the wage distribution due to the minimum wage. The gap then rises to a high of about .35 at the 30th percentile and falls thereafter. There is no strong evidence of a 'glass ceiling', in that blacks do not appear to be disproportionately excluded from high paying jobs. ${ }^{12}$ To test whether these differentials are significantly different, we estimated quantile regressions of log wages on race for each of the deciles. The estimation procedure used provides an estimate of the entire variance-covariance matrix of the system by bootstrapping. This is used to test restrictions across equations. The null hypothesis of equal differentials at all deciles was rejected with a p-value close to zero.

Comparing ww with bb also allows us to look at issues of low-pay for the black and white populations respectively. Traditionally thresholds for low pay are based on some transformation of the median wage. To illustrate the importance of a distributional approach for policy analysis, we choose an arbitrary low pay threshold of $3 / 4$ of the median wage for the entire sample. This threshold is represented by the vertical line in Fig. $1 .^{13}$ Based on the estimated densities, we find that approximately $19 \%$ of blacks are classified as low-paid with our cutoff as compared to just 7\% of whites.

Neal and Johnson argue that controlling for differences in AFQT scores can explain most of the average black-white wage gap. Our semi-parametric approach allows us to decompose the average wage gap using less restrictive parametric assumptions. The semiparametric results indicate that the average log wage gap falls from .26 to $.07 .{ }^{14}$ Thus differences in test scores account for approximately $73 \%$ of the average wage gap. The results for average wages based on the semi-parametric approach are almost identical to

\footnotetext{
12 For a discussion of this topic in relation to gender wage differentials see Albrecht et al. (2003).

13 This threshold is used for illustrative purposes. Similar results are obtained for a range of other possible thresholds.

${ }^{14}$ Formally the semiparametic estimates of the mean effects are $\hat{\mu}_{w}-\hat{\mu}_{B}$ and $\hat{\mu}_{w}-\hat{\mu}_{B W}$ respectively. The estimates for $\mu_{W}, \mu_{B}$ and $\mu_{B W}$ are based on the estimated densities $\hat{f}_{W W}, \hat{f}_{B B}, \hat{f}_{B W}$ respectively.
} 


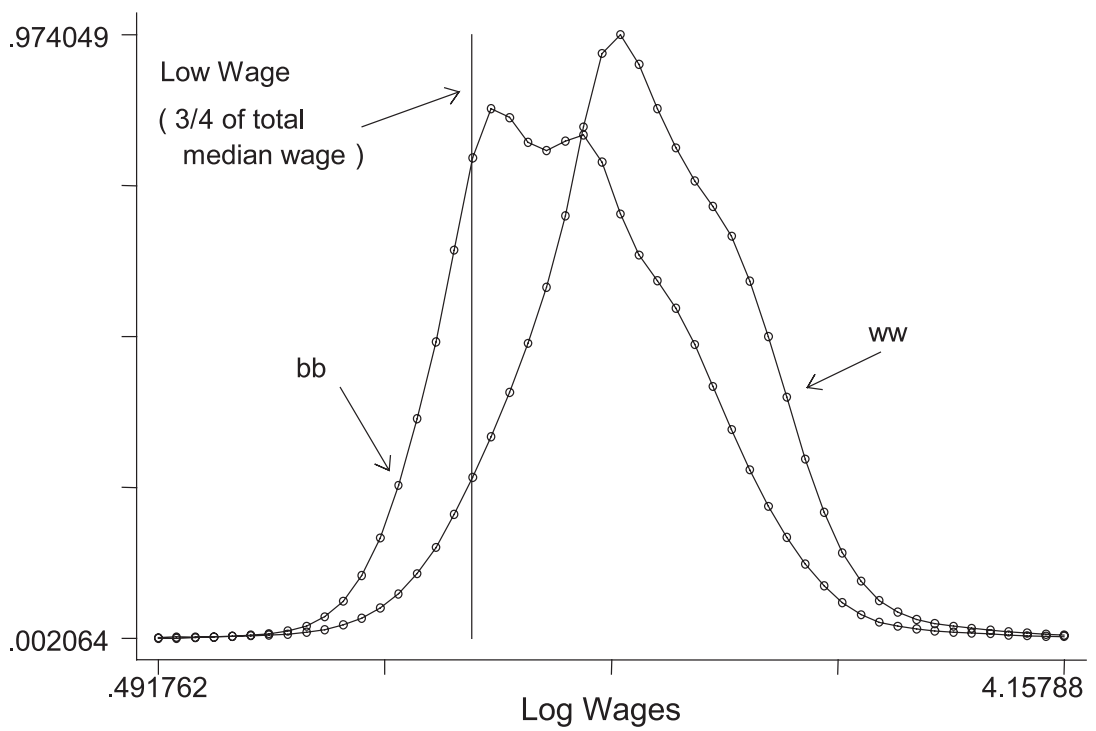

Fig. 1. Black and white log wage densities.

those presented by Neal and Johnson using the linear regression model. Therefore the interesting contribution of the semi-parametric approach for this paper is the ability to analyse wage differences at other parts of the distribution.

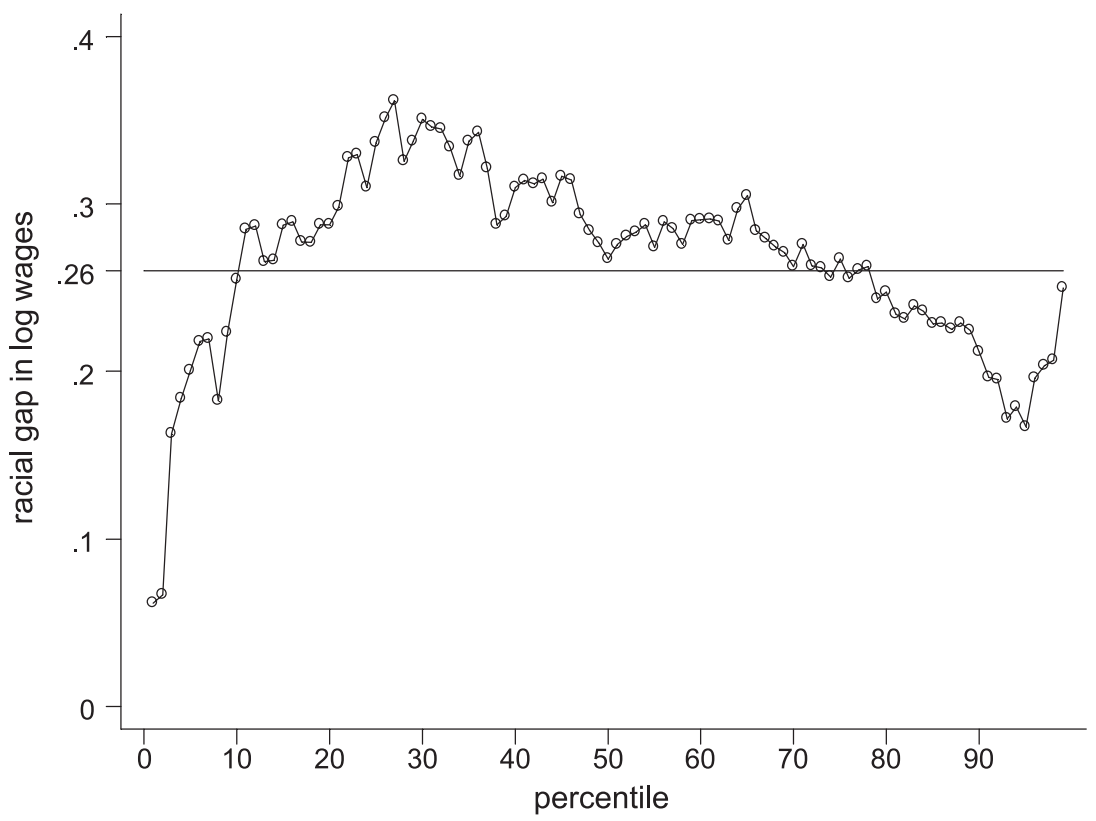

Fig. 2. Racial log wage gap at each centile of the distribution. 
As noted earlier there is no reason to believe, a-priori, that the same forces affect the wage gap for both low and high wage workers. We use the procedure outlined above to estimate the density of wages for a group of workers who are paid according to the black wage structure and are like blacks in all other respects except they have the white distribution of test scores. A key element of this approach is the estimated reweighting function $\hat{\psi}\left(t_{i}\right)$. The purpose of these weights is to try and match the skill distributions of black and white workers in the sample. In order for the procedure to be meaningful the counterfactual density of test scores for black workers, that is the density of black test scores estimated using weighted kernel procedures with weights $\hat{\psi}\left(t_{i}\right)$, should resemble the raw density of test scores for white workers. To evaluate the success of the weighting function Fig. 3 provides kernel density estimates of the skill distributions for black and white workers, along with the counterfactual density. Looking at the raw distributions, we clearly see substantial differences between the black and white test score densities. To the extent that our reweighting procedure is successful we would expect the counterfactual test score density for black workers to be much closer to the raw white test score density. As expected the graph shows that this is the case. The similarity of these two densities confirms that the weighting scheme used in this paper performs well in matching the black distribution of tests scores to the white population.

Our results for the densities of log wages are presented in Fig. 4. Again bb denotes the density of log wages for black workers and ww denotes the white density. bw denotes the counterfactual density, namely the density of log wages for workers with the white distribution of test scores but paid according to the black wage structure. At the upper end of the wage distribution the densities ww and bw coincide. The proportion of high wage people is very similar for both the ww and bw densities. For instance, the proportion of

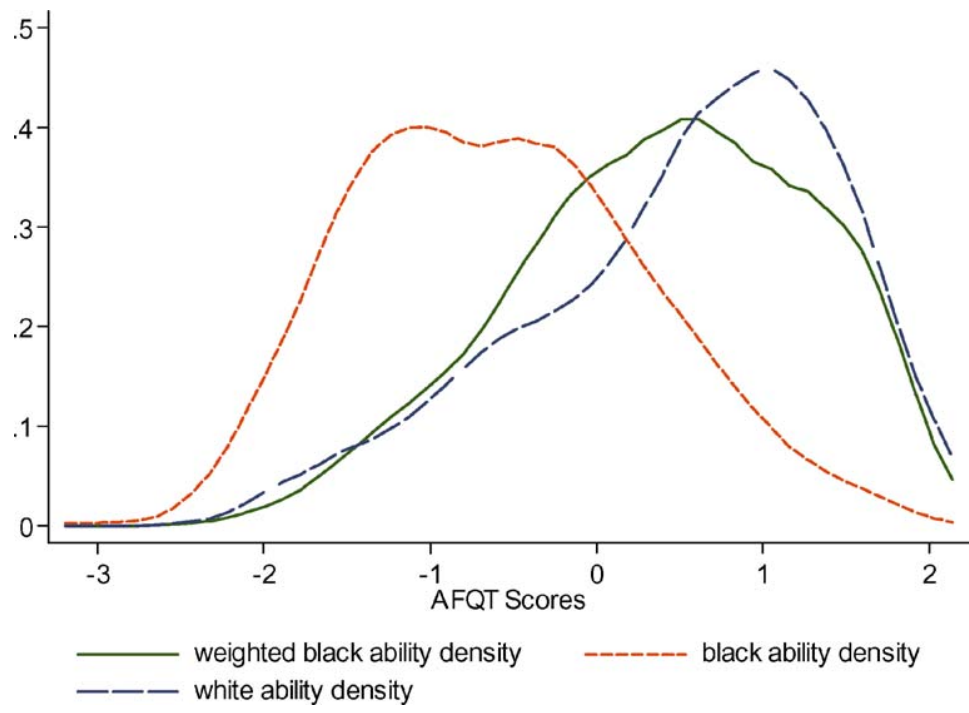

Fig. 3. Distribution of test scores. 


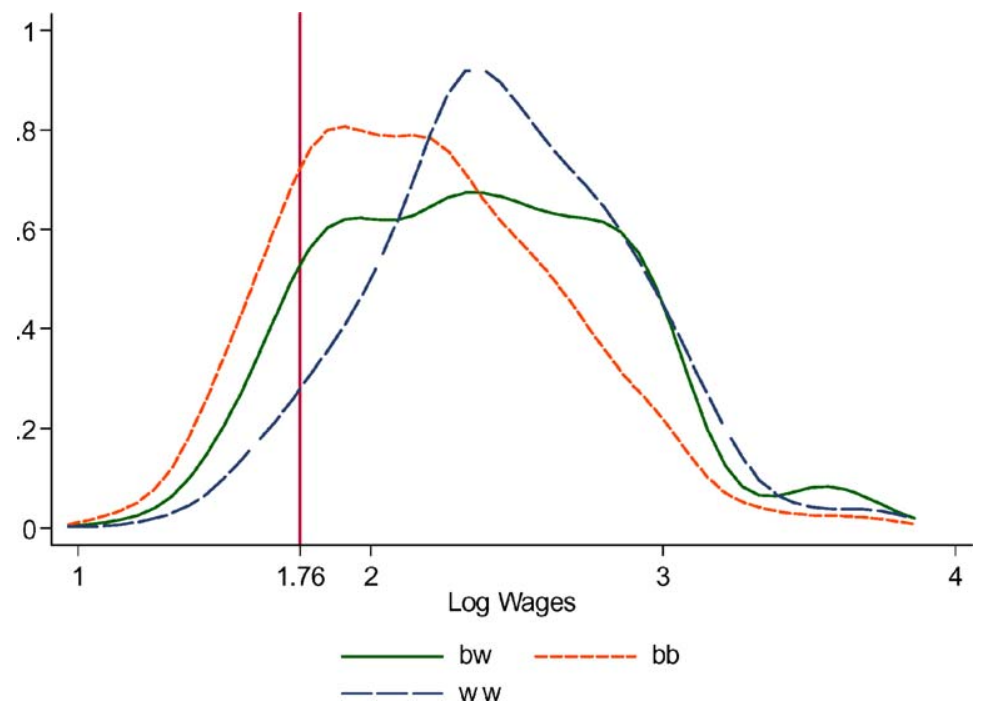

Fig. 4. Log wage densities for black workers and white workers and the counterfactual log wage density for black workers with white skills.

workers earning more than the 75 th percentile of the combined wage distribution is $31 \%$ and $30 \%$ for the white workers (ww) and the reweighted black sample (bw) respectively. However this is not the case for low wage workers. The proportion of low paid workers calculated using the bw density (approximately 12\%) is still substantially higher than that based on the ww density (approximately 7\%). These differences reflect differences in the wage structure between black and white workers and differences in characteristics other than test scores.

Table 3 highlights the unequal effect of test scores throughout the distribution. The first column summarises the results in Fig. 2 and shows the raw log wage gap at each of the percentiles, $\left[F_{W W}^{-1}(p)-F_{B B}^{-1}(p)\right]$. The second column shows the proportion of this wage

Table 3

The proportion of the black-white log wage gap due to test scores at different parts of the distribution

\begin{tabular}{|c|c|c|c|}
\hline \multirow[t]{2}{*}{ Wage Percentile } & \multirow{2}{*}{$\frac{\text { Raw Log Wage Gap }}{\left(\mathrm{F}_{\mathrm{WW}}{ }^{-1}(\mathrm{p})-\mathrm{F}_{\mathrm{BB}}^{-1}(\mathrm{p})\right)}$} & \multirow{2}{*}{$\frac{\text { Proportion due to Test Scores }}{\text { PropTest (p) }}$} & \multirow[t]{2}{*}{$90 \%$ confidence interval } \\
\hline & & & \\
\hline 10th Percentile & .263 & .43 & {$[.27-.64]$} \\
\hline 20th Percentile & .287 & .41 & {$[.24-.62]$} \\
\hline 30th Percentile & .351 & .49 & {$[.28-.74]$} \\
\hline 40th Percentile & .310 & .61 & {$[.36-.87]$} \\
\hline 50th Percentile & .267 & .72 & {$[.46-1.02]$} \\
\hline 60th Percentile & .291 & .82 & {$[.52-1.16]$} \\
\hline 70th Percentile & .265 & .90 & {$[.57-1.28]$} \\
\hline 80th Percentile & .250 & .94 & {$[.58-1.31]$} \\
\hline 90th Percentile & .211 & .88 & {$[.52-1.49]$} \\
\hline
\end{tabular}

\footnotetext{
a These confidence intervals were estimated using a bootstrapping procedure with 1000 replications.
} 
gap that is due to test scores at each decile of the distribution. We denote this as PropTest (p) and calculate it as follows:

$$
\operatorname{PropTest}(p)=\frac{\left[F_{B W}^{-1}(p)-F_{B B}^{-1}(p)\right]}{\left[F_{W W}^{-1}(p)-F_{B B}^{-1}(p)\right]}
$$

The proportion of the wage gap explained rises from about $40 \%$ at the second decile to approximately $95 \%$ at the top two deciles. The final column in Table 3 provides $90 \%$ confidence intervals for the proportion explained by test scores. We calculate these confidence intervals using bootstrapping techniques. To construct these intervals we estimated the proportions described above for 1000 different data sets drawn with replacement from the original data. This in turn provides a distribution of estimates for the proportion of the wage gap explained at each of the deciles. These empirical distributions were used to calculate the confidence intervals reported in Table 3. Unfortunately the confidence intervals constructed in this fashion are such that there is a small overlap between the intervals for the lower deciles and those for the upper deciles. As a result we just fail to reject the hypothesis that the proportion of the wage gap explained at each decile is equal at the 10 percent significance level. This reflects the structure of the data and the nature of the policy experiment under consideration. The experiment of providing black workers with the white skill levels requires that there are sufficient black workers with skill levels comparable to whites in order to identify the effect. Unfortunately there are relatively few blacks with high test scores. We have also considered the alternative counterfactual of providing white workers with the black distribution of skills. Identification in this experiment is achieved using those white workers with relatively low test scores. The distribution of the ability scores is such that this latter counterfactual is more precisely

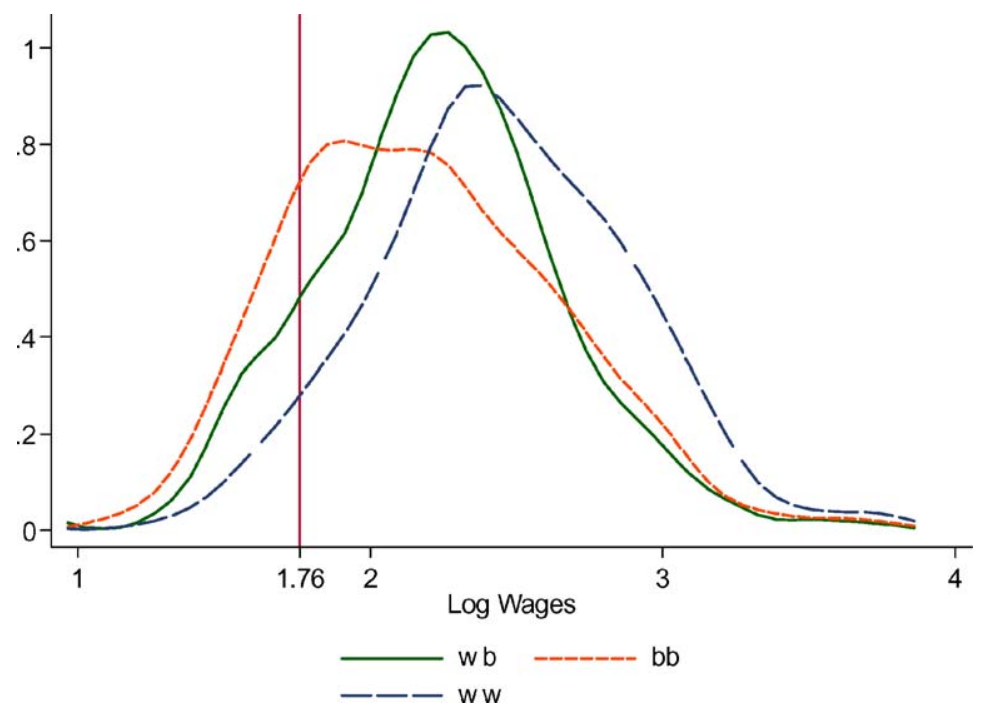

Fig. 5. Log wage density for black workers and white workers and the counterfactual log wage density for white workers with black skills. 
Table 4

The proportion of the black-white log wage gap due to test scores and other factors throughout the distribution

\begin{tabular}{lcc}
\hline Wage Percentile & $\begin{array}{l}\text { Proportion due } \\
\text { to Test Scores }\end{array}$ & $\begin{array}{c}\text { Proportion due to Test Scores } \\
\text { and other characteristics }\end{array}$ \\
\hline 10th Percentile & .51 & .51 \\
20th Percentile & .46 & .47 \\
30th Percentile & .54 & .56 \\
40th Percentile & .68 & .73 \\
50th Percentile & .83 & .91 \\
60th Percentile & .99 & 1.04 \\
70th Percentile & 1.12 & 1.11 \\
80th Percentile & 1.14 & 1.10 \\
90th Percentile & 1.06 & 1.00 \\
\hline
\end{tabular}

${ }^{a}$ The other characteristics included in this specification include the Rotter and Rosen measures of non-cognitive skills and father's education.

estimated. ${ }^{15}$ Qualitatively the results of this thought experiment are very similar to those presented earlier. The results for this counterfactual are presented in Fig. 5 and show that adjusting the distribution of test scores explains more of the wage gap for black workers above the median than for workers below it. Furthermore the differences above and below the median in the amount explained are now statistically significantly different. However in the context of a public policy debate this latter experiment is not a reasonable alternative. For this reason we decided to concentrate on the experiment that provides black workers with the white distribution of skills while recognising that more data would be desirable in order to get more precise estimates.

Neal and Johnson draw attention to the importance of test scores in accounting for the average black-white wage gap. Using a semi-parametric procedure to estimate counterfactual wage densities, we have shown that there is still a significant role for the residual component in explaining wage differences among the most disadvantaged black workers. In order to identify the sources of these remaining differences, we examine the impact of controlling for measures of non-cognitive skills and family characteristics. ${ }^{16}$ Heckman and Rubinstein (2001) state that "much of the effectiveness of early-childhood interventions comes in boosting non-cognitive skills and fostering motivation." Given the quantitative importance of non-cognitive skills they argue "that social policy should be more active in attempting to alter them, especially for children from disadvantaged backgrounds.” To examine the extent to which these factors can explain the residual gap among low wage black workers, we include two widely used measures of non-cognitive skills. The measures that we use are the Rotter Locus of Control scale developed by Rotter (1966) and the Rosenberg Self-Esteem Scale (Rosenberg (1965)) which we discussed earlier in the paper. We use the level of father's education to proxy for family background. The results are presented in Table 4. Including these variables in the analysis lowers the sample size

\footnotetext{
${ }^{15}$ We had 50 observations on white workers with test scores in the bottom quartile of the black distribution but only 12 observations on black workers with test scores in the top quartile of the white distribution.

${ }^{16}$ The NLSY also contains some interesting variables on the type of schools attended by the respondents that we would liked to have used in our analysis. However including these measures reduced the sample sizes too much.
} 
somewhat, so for ease of comparison the first column presents the counterfactual based on test scores only, for this smaller sample. The trend is very much in keeping with the results presented in Table 3. The second column presents the results when black characteristics are also adjusted for measures of non-cognitive skills and our proxy for family background. While these characteristics are important in explaining individual wages ${ }^{17}$, they have very little additional impact on the black-white wage gap at any part of the distribution once we have controlled for differences in cognitive skills. Identifying the source of the remaining wage gap for disadvantaged black workers is important if we are to further improve circumstances for low-paid black workers.

\section{Conclusion}

This paper extends the work of Neal and Johnson by using a semi-parametric approach to examine the impact of cognitive skills on the black-white wage gap. Recently researchers have argued that the disadvantages facing young black workers arise from obstacles they faced as children in acquiring human capital and that public policy should focus on the plight of these children in acquiring skills that are valued in the labor market. In keeping with these studies, we find that differences in AFQT scores are an important determinant of the wage gap throughout the distribution. However our results indicate that a substantial unexplained component remains for disadvantaged black workers. Including measures of non-cognitive skill and family background had little additional impact on the wage gap for these workers. Further work is still needed in order to understand more fully the obstacles faced by the most disadvantaged black workers.

\section{Acknowledgement}

We thank participants at the Dublin Labour Studies Group Workshop, the European Society of Population Economics Annual Conference, the European Association of Labour Economists Conference, the Irish Economic Association annual conference, seminar participants at NUI Cork and the ESRI and Luc Hens for helpful comments on an earlier version of this paper. We would also like to acknowledge the helpful comments of an anonymous referee. The third author acknowledges financial support from the Interuniversity Attraction Poles Program-Belgian Science Policy, Contract No. P5/21.

\section{References}

Albrecht, J., Bjorklund, A., Vroman, S., 2003. Is there a glass ceiling in Sweden? Journal of Labor Economics 21 (1), $145-177$.

Altonji, J., Blank, R., 1999. In: Ashenfelter, O., Card, D. (Eds.), Race and Gender in the Labor Market, vol. 3C. Elsevier Science Publishers.

\footnotetext{
${ }^{17}$ In a regression of wages on characteristics the measures of non-cognitive skills and family background all show up as being statistically significant with the expected sign.
} 
Barsky, R., Bound, J., Charles, K., Lupton, J., 2002. Accounting for the black-white wealth gap: a non-parametric approach. Journal of the American Statistical Association 97 (459), 663-673.

Blinder, A., 1973. Wage discrimination: reduced form and structural variables. Journal of Human Resources 8 (4), 436-455.

Bonjour, D., Gerfin, M., 2001. The unequal distribution of unequal pay: an empirical analysis of the gender wage gap in Switzerland. Empirical Economics 26 (2), 407-427.

Cawley, J., Conneely, K., Heckman, J., Vytlacil, E., 1997. In: Devlin, B., Fienberg, S., Resnick, D., Roeder, K. (Eds.), Cognitive Ability, Wages and Meritocracy. Springer Verlag, New York, pp. 179-192.

Darity, W.A., Mason, P.L., 1998. Evidence on discrimination in employment: codes of color, codes of gender. Journal of Economic Perspectives 12 (2), 63-90.

DiNardo, J., Tobias, J., 2001. Nonparametric density and regression estimation. The Journal of Economic Perspectives 15 (4), $11-28$.

DiNardo, J., Fortin, N., Lemieux, T., 1996. Labor market institutions and the distribution of wages 1973-1992: a semi-parametric approach. Econometrica 64 (5), 1001-1044.

Dunfion, R., Duncan, G., Brooks-Gunn, J., 2001. As ye sweep so shall ye reap. The American Economic Review 91 (2), $150-154$.

Gardeazabal, J., Ugidos, A., 2004. Gender discrimination at quantiles. Journal of Population Economics (forthcoming).

Heckman, J., 1998. Detecting discrimination. Journal of Economic Perspectives 12 (2), 101-116.

Heckman, J., Rubinstein, Y., 2001. The importance of noncognitive skills: lessons from the GED testing program. The American Economic Review 91 (2), 145-149.

Jenkins, S.P., 1994. Earnings discrimination measurement: a distributional approach. Journal of Econometrics 61 (2), $81-102$.

Neal, D.A., Johnson, W.R., 1996. The role of premarket factors in black-white wage differences. Journal of Political Economy 104 (5), 869-895.

Oaxaca, R., 1973. Male-female wage differentials in urban labor markets. International Economic Review 14 (3), $693-709$.

Pergamit, M.R., Pierret, C.R., Rothstein, D.S., Veum, J.T., 2001. Data watch: the national longitudinal surveys. Journal of Economic Perspectives 15 (2), 239-253.

Rodgers, W., Spriggs, W.E., 1996. What does AFQT really measure?: race, wages, schooling and the AFQT score. The Review of Black Political Economy 24 (4), 13-46.

Roemer, J., 1998. Equality of Opportunity. Harvard University Press, Cambridge, MA.

Rosenberg, M., 1965. Society and the Adolescent Self-Image. Princeton University Press, New Jersey.

Rotter, J., 1966. Generalized expectancies for internal versus external control of reinforcement. Psychological Monographs 80 (609), 1-28.

Silverman, B.W., 1986. Density Estimation for Statistics and Data Analysis. Chapman and Hall, London. 